\title{
POLAR CAP ACCRETION ONTO MAGNETIZED NEUTRON STARS: AN ANALYTIC SOLUTION
}

\author{
Jonathan Arons* and Richard I. Klein* \\ Department of Astronomy, University of California \\ at Berkeley, and Institute of Geophysics and \\ Planetary Physics, University of California, \\ Lawrence Livermore Laboratory
}

This abstract should be read in conjunction with the papers by Arons and by Klein and Arons in these proceedings. In the context of the accretion models described there, one can find an analytic solution for the flow down the polar field lines if a number of simplifying assumptions are made. These are (1) steady flow in the co-rotating frame; (2) radiation pressure large compared to gas pressure; (3) pure scattering for the Rosseland opacity, with the magnetic corrections set equal to constants instead of using the actual functions of temperature; (4) diffusion flux of radiative energy proportional to the gradient of the energy density alone, instead of the correct sum of terms proportional to the photon energy density and the number density gradients; and (5) below a radiative shock, subsonic flow in approximate hydrostatic equilibrium. We assumed dipole geometry, and also assume the mass flux is independent of distance from the magnetic axis. The essential trick is to use (1), (2) and (5) to write the advective contribution to the radiation transfer equation as $\dot{\mathrm{Mg}} /$ area $=$ rate at which gravity does work on a fluid element, and use (3) and (4) to write the nonlinear diffusion flux as the ratio of gradients in the energy density. Then the multidimensional diffusion equation can be cast in a separable, linear form by using the logarithmic radial gradient of the energy density as the basic variable (see also Kirk, J., 1985, Astron. and Astrophys., 142, 430). The result is exponential stratification of the energy density, velocity and mass density along $B$ with scale height $\mathrm{R}_{*}\left[\mathrm{~L}_{\text {(eff }}\right.$ (ef $\left./ 4 \mathrm{~L}_{\text {cos }}\right]$; the effective Eddington luminosity is discussed by Arons, these proceedings. This result can be understood as the result of almost exact balance between upward diffusion and downward advection of photons in the optically thick medium. The same fluid quantities are stratified in a Gaussian manner across $B$, with angular half width at half maximum $\Delta \theta=$ $\left[\mathrm{L}(\mathrm{eff}) / \mathrm{L}_{\text {cap }}\right]\left(\mathrm{r} / \mathrm{R}_{*}\right)^{3 / 2}$. These distributions agree well with more sophisticated computational results, during times when the flow is steady. When used as a basis for calculations of the radiative entropy, the calculated emergent spectra are not dissimilar to the spectra of high luminosity, accretion powered pulsars.

\footnotetext{
*also at Department of Physics, University of California at Berkeley \#also at Lawrence Livermore Laboratory
} 